\title{
'A study correlating the quantity and duration of alcohol consumption with liver function tests'
}

\author{
Dr. Walter A, Dr. Mohammed Ashraf. \\ Father Muller Medical College Hospital, Kankanady, Mangalore, Karanataka, India
}

\begin{abstract}
Objectives:Alcoholism is an important health problem all over the world. Biochemical tests are important tools in diagnosing alcohol abuse and identifying the stages of alcoholic liver disease. This study is an attempt to describe the biochemical changes that can be observed in individuals with history of significant alcohol intake as well as to correlate the quantity and duration of alcohol consumption with derangements in the liver function tests.

Methods:Fifty males with history of alcohol consumption in significant quantity were included in the study and were interviewed for the quantity and duration of alcohol consumption. Liver function tests which included estimation of serum albumin, globulin, AST, ALT, prothrombin time and bilirubin were done. Derangements in liver function tests were observed and correlated with the quantity and duration of alcohol consumption.

Results: Of the 50 individuals, 40\% had hypoalbuminemia, 28\% had hyperglobulinemia; the percentage of patients with elevated AST,ALT, ALP, bilirubin and prolonged prothrombin time were $42 \%, 8 \%, 12 \%, 40 \%$ and $28 \%$ respectively. $76 \%$ had ratio of AST to ALT more than 1 with $40 \%$ having more than 2. Karl-Pearson correlation correlation for quantity of alcohol consumption with albumin, blumin, SGPT, bilirubin and prothrombin time was $-0.497,0.519,0.440,0.354$ and 0.380 with $p$ value $0.00,0.00,0.001,0.012$ and 0.007 respectively. The ' $r$ ' value for duration of alcohol with albumin and bilirubin was - 0.485 and 0.486 with a $p$ value of 0.00 and 0.00 respectively.

Conclusion: There was a statistically significant positive correlation between quantity of alcohol consumption and serum levels of globulin, SGPT, bilirubin and prothrombin time. Where as for serum albumin levels there was a negative correlation. There was a statistically significant correlation between duration of alcohol consumption with albumin, which is negative and bilirubin, positively.
\end{abstract}

Keywords: Alcoholism, alcoholic liver disease, liver function tests

\section{Introduction}

Alcohol consumption has been a part of our culture from many centuries. It is the most common addictive substance available in the present world and has become an essential part of our existence in all age groups and sexes ${ }^{1}$. Alcohol consumption in various degrees, be it binge drinking or choronic consumption, or even moderate drinking in inappropriate circumstances posed a threat to the health of the individual as well to the family, friends and society through anti-social behavior, crime, unemployment and loss of productivity.

Alcohol consumption is responsible for 3.8\% of global mortalityand $4.6 \%$ of disability-adjusted life-years (DALYs) lost due to premature death. The attributable burden in Europe, with $6.5 \%$ of all deaths and $11.6 \%$ of DALYs attributable to alcohol, is the highest proportion of total ill health and premature deaths due to alcohol of all WHO regions ${ }^{2}$.

Alcohol causes various health problems by affecting various organs in the body, such as liver, pancreas and central nervous system. Liver being the organ where alcohol is metabolized, it suffers the greatest insult by alcohol. Alcohol being a foreign substance and potentially toxic, liver cells sense it to be a poisonous substance, metabolises and detoxifies it. Alcohol causes liver cell damage by various mechanisms, some directly by the metabolites and others by the byproducts of metabolism such as free oxygen radicals. The exact mechanism by which alcohol causes liver cell injury is not completely understood but the role of various factors are hypothesized such as free radicals, hypoxia, antioxidants, eicosanoids, cytokines, endotoxin, adduct formation, inflammatory agents and fibrosis. Liver disease caused by alcohol can progress through the stages of steatosis, inflammation, necrosis, fibrosis and cirrhosis- leading to fatty liver, alcoholic hepatitis, cirrhosis of liver and rarely hepatocellular carcinoma ${ }^{3}$.

The liver has various functions such as storage, synthetic and detoxification. There are biochemical tests available to assess these aspects of liver function. In the diseased state liver function tests may be deranged and in alcohol consumption also there are few derangements in the liver functions. This study is an attempt to observe and describe the derangements in liver functions biochemically, depending on the quantity and duration of alcohol consumption. 


\section{Methods}

The study was conducted in Father Muller Medical College Hospital (FMMCH), Mangalore, Karnataka over a period of three months from August 2013 to October 2013.

\section{Source of data:}

Patients admitted in FMMCH with various health problems and with history of alcohol consumption from August 2013 to October 2013.

\section{Method of collection of data:}

50 male patients were included in the study who had history of drinking alcohol in significant quantity.

\section{Inclusion criteria:}

1. Patients aged above 18 years

2. Male gender

3. History of drinking alcohol to a minimum of $60 \mathrm{~g}$ per day

\section{Exclusion criteria:}

1. Patients diagnosed to have non-alcoholic liver diseases

2. Patients with history of consuming hepatotoxic drugs in the recent past

3. Patients diagnosed with malaria, dengue fever, viral hepatitis which can by itself derange liver enzymes

4. Patients with septicemia

5. Patients diagnosed to have congestive heart failure, right sided heart failure or other causes of

\section{Study design:} congestive /ischemic hepatitis.

Patients were interviewed for history of alcohol consumption in significant quantity and those with a minimum alcohol intake of $60 \mathrm{~g}$ per day were included in the study. Details of the presenting complaints, comorbidities, history of recent ailments, drug history, duration and quantity of alcohol intake, history of any other substance abuse were documented. Clinical examination was done in detail in particular relation to alcoholic liver disease.

The following laboratory investigations were noted in relation to liver functions:

- Serum albumin

- Globulin

- Aspartate aminotransferase (AST/SGOT)

- Alanine aminotransferase (ALT/SGPT)

- Prothrombin time

- Total bilirubin

- Direct bilirubin

- Indirect bilirubin

\section{Study type:}

Decriptive cross-sectional study.

\section{Data analysis:}

Data was tabulated and analysed by frequency, mean, percentage and Karl- Pearson correlation coefficient.

Total of 50 male patients were included in the study.

\section{Results}

Age Distribution Of The Study Group:

Table 1: Age distribution of the study group

\begin{tabular}{|l|l|l|}
\hline Age in years & Percentage & n \\
\hline $20-29$ & 4 & 2 \\
\hline $30-39$ & 32 & 16 \\
\hline $40-49$ & 36 & 18 \\
\hline $50-59$ & 24 & 12 \\
\hline$>60$ & 4 & 2 \\
\hline
\end{tabular}

Majority were in the age group of 40-49 years (36\%). 32\% of the patients belonged to the age group of 30-39 years. $24 \%$ of the patients belonged to the age group of 50-59 years. Only $4 \%$ each were in the age group of 2030 years and $>60$ years. 


\section{Quantity Of Alcohol Consumption Of The Study Group}

Table 2: Quantity of alcohol distribution of the study group

\begin{tabular}{|l|l|l|}
\hline $\begin{array}{l}\text { Grams of alcohol per } \\
\text { day }\end{array}$ & Percentage & n \\
\hline 60 & 40 & 20 \\
\hline $61-70$ & 16 & 8 \\
\hline $71-80$ & 12 & 6 \\
\hline $81-90$ & 20 & 10 \\
\hline$>90$ & 12 & 6 \\
\hline
\end{tabular}

In the present study majority of the patient, i.e. $40 \%$ of the subjects were consuming $60 \mathrm{~g}$ of alcohol per day. This followed by the $28 \%$ of the subjects consuming $81-90 \mathrm{~g}$ per day, $16 \%$ of the subjects consuming $61-70$ $\mathrm{g}$ of alcohol per day.

\section{Duration Of Alcohol Consumption Of The Study Group}

Table 3: Distribution of the study group based on the duration of alcohol consumption

\begin{tabular}{|l|l|l|}
\hline Years & Percentage & $\mathbf{n}$ \\
\hline 10 to 12 & 26 & 13 \\
\hline 12 t0 14 & 34 & 17 \\
\hline $14-16$ & 6 & 3 \\
\hline $16-18$ & 6 & 3 \\
\hline $18-20$ & 6 & 3 \\
\hline $20-22$ & 14 & 7 \\
\hline$>22$ & 8 & 4 \\
\hline
\end{tabular}

In the study group $60 \%$ of the patients were consuming for a period of $10-14$ years and $40 \%$ of the subjects were consuming for a period more than 14 years.

Sgot /Sgpt Ratio In The Study Population

Table 4: Ratio of SGOT and SGPT in the study group

\begin{tabular}{|l|l|l|}
\hline SGOT/SGPT & Percentage & $\mathbf{n}$ \\
\hline$<1$ & 24 & 12 \\
\hline 1 TO 2 & 36 & 18 \\
\hline$>2$ & 40 & 20 \\
\hline
\end{tabular}

It was observed in the study that most of the patients (40\%) had a SGOT/ SGPT of more than 2. This was followed by $36 \%$ of the patients having SGOT/SGPT ranging between 1 and 2 . Only $24 \%$ of the patients had SGOT/SGPT below 1.

\section{Correlations Of The Quantity And Duration Of Alcohol With The Liver Function Tests} Pearson correlations:

Table 5: Quantity of alcohol and liver function tests

\begin{tabular}{|l|l|l|}
\hline Albumin & r & p \\
\hline Globulin & -0.497 & 0.00 \\
\hline Aspartate aminotransferase & 0.519 & 0.00 \\
\hline Alanine aminotransferase & 0.216 & 0.132 \\
\hline Alkaline phosphatase & 0.44 & 0.001 \\
\hline Prothrombin time & 0.196 & 0.174 \\
\hline Bilirubin & 0.38 & 0.007 \\
\hline
\end{tabular}


Table 6: Duration of alcohol consumption and liver function tests

\begin{tabular}{|l|l|l|}
\hline & $\mathbf{r}$ & $\mathbf{p}$ \\
\hline Albumin & -0.485 & 0.00 \\
\hline Globulin & 0.362 & 0.10 \\
\hline Aspartate aminotransferase & 0.086 & 0.551 \\
\hline Alanine aminotransferase & 0.008 & 0.958 \\
\hline Alkaline phosphatase & -0.113 & 0.435 \\
\hline Prothrombin time & 0.084 & 0.56 \\
\hline Bilirubin & 0.486 & 0.00 \\
\hline
\end{tabular}

There appears to be a negative correlation of quantity as well as duration of alcohol consumption with serum albumin of the subjects in the study group. There is a positive correlation between quantity of alcohol consumed per day with serum globulin, SGPT, PT and serum bilirubin. There is also a positive correlation between duration of alcohol consumption with serum bilirubin in the study group.

\section{Discussion}

Alcohol consumption causes changes in the liver functions both in acute consumption as well as chronic consumption. In the present study significant changes were detected biochemically based on the liver function tests mainly using the parameters- serum albumin, globulin, SGOT, SGPT, ALP, PT and bilirubin levels.

\section{Effects of alcohol on the albumin and globulin levels:}

In the present study, $40 \%$ of the subjects were observed to have hypoalbuminemia. There was a statistically significant negative correlation between the quantity of alcohol consumed and serum albumin levels of patients in the study. There was also a statistically significant negative correlation between duration of history of alcohol and serum albumin levels.

Hyperglobulinemia was observed in $28 \%$ of the patients. There was a significant positive correlation between the quantity of alcohol consumption and serum globulin levels in the study group.

Usharani $\mathrm{B}$ et $\mathrm{al}^{4}$ in their study observed no significant alteration in the total proteins, albumin and globulin in the group which had alcohol consumption history for the past 6 to 15 years but in the groups with alcohol history longer than 15 years the serum concentration of albumin was significantly decrease and that of globulin was significantly elevated in comparison to the control group. Similar results were also observed by Das SK et $\mathrm{al}^{5}$ in their study.

\section{Effects of alcohol on hepatic enzymes:}

SGOT and SGPT are intracellular enzymes which are released on cell injury. Thus these are indicators of liver cell injury even in cases of alcohol mediated liver cell injury. It is observed that these transaminases can increase upto a level of $300 \mathrm{IU} / \mathrm{L}$ but levels beyond this are usually indicative of other causes of liver cell injuru such as viral hepatitis. In cases of alcohol induced liver damage it is observed that the ratio of SGOT to SGPT is usually more than 1 and if it is more than $2,90 \%$ of the cases are due to alcohol ${ }^{6}$.

In the present study $42 \%$ of the subjects had elevated SGOT levels and $8 \%$ of the subjects had elevated SGPT levels. The SGOT to SGPT ratio in the study group was more than 2 in $40 \%$ of the subjects, 1 to 2 in $36 \%$ of the subjects and $24 \%$ of the subjects had the ratio less than 1 . There was a a statistically significant correlation between quantity of alcohol consumption and SGPT levels. Serum ALP level was elevated in 12\% of the subjects in the study group.

Leena Waes et al in their study reported 50\% of the patients to have elevated SGOT levels and $57 \%$ of the patients had elevated SGPT levels ${ }^{7}$.

Usharani B et $\mathrm{al}^{4}$ reported in their study that in the group consuming alcohol for the past 6-15 years there was no significant changes in the AST and ALT levels where as the the activity of ALP was elevated in comparison to the control group. In the group consuming alcohol for the past 16-25 years there was no significant elevation I the levels of AST but there was significant elevations in the ALT levels. Whereas in the group with alcohol consumption for more than 25 years all the hepatic marker enzymes were significantly elevated.

Das SK et $\mathrm{al}^{5}$ in their study observed a significant increase in the activity of AST and ALT in the patients with history of alcohol consumption in caomparison eith the control group and also observed a greater AST to ALT ratio in the patients with alcohol consumption history. They also observed significant elevation in the serum ALP levels in the patients with alcohol consumption history. 


\section{Effects of alcohol on PT:}

In the present study $28 \%$ of the patients had prolonged prothrombin time. Prolonged prothrombin time suggests that the synthetic function of the liver with regard to clotting factors is affected. Prolonged prothrombin time rules out fatty liver alone as the diagnosis. There was a statistically significant positive correlation between the quantity of alcohol consumption and prothrombin time.

Madenhall ${ }^{8}$ in his study observed that prothrombin time was prolonged in $65 \%$ of the patients with alcohol hepatitis and $90 \%$ of the patients with cirrhosis.

\section{Effect of alcohol on serum bilirubin levels:}

Hyperbilirubinemia was observed in $40 \%$ of the patients. There was a statistically significant positive correlation between the quantity and duration of alcohol consumption with serum bilirubin level.

Das SK et al ${ }^{5}$ in their study observed that in patients with history of alcohol consumption the elevation of serum conjugated bilirubin was 2-7 times but the elevation of serum unconjugated bilirubin was far less in the same population in comparison to the healthy volunteers.

In the study conducted by Usharani B et $\mathrm{al}^{4}$, hyperbilirubinemia was observed only in those subjects with history of more than 25 years of alcohol consumption and there was no hyperbilirubinemia in those with history of alcohol consumption of less than 25 years.

In the Medenhall study hyperbilirubinemia was observed in $65 \%$ of the patients with alcoholic hepatitis and in those with cirrhosis was $90 \%$.

\section{Limitations of the study:}

1. Stratification of the patients into those with alcoholic fatty liver, alcoholic hepatitis and cirrhosis based on ultrasonogram of the abdomen and histopathological examination and then correlating with the biochemical parameters may have yielded more information.

2. Other parameters which are used biochemically to assess the liver function in alcoholics such as GGT, $\mathrm{MCV}$, lipids were not assessed

\section{Conclusion:}

Alcohol consumption causes varying degree of liver cell injury which can be assessed biochemically by the liver function tests. Alcohol consumption results in hypoalbuminemia, hypergammaglobulinemia; elevation of hepatic marker enzymes such as ALT, AST, Alkaline phosphatase; prolonged prothrombin time and hyperbilirubinemia. There is a statistically significant positive correlation between quantity of alcohol consumption and serum levels of globulin, SGPT, bilirubin and prothrombin time; whereas with serum albumin levels there was a negative correlation. There was a statistically significant negative correlation between duration of alcohol consumption with albumin and with bilirubin levels there was a positive correlation indicating consumption of alcohol over a prolonged period results in hypoalbuminemia and hyperbilirubinemia.

\section{Summary}

1. The present study was a descriptive cross sectional study which included 50 male patients who had history of significant alcohol intake.

2. The aim of the study was to describe the changes in the liver functions biochemically and to see if there is a correlation between the quantity and duration of alcohol consumption with the derangements in the liver function tests.

3. It was observed in the study that $40 \%$ of the subjects were consuming $60 \mathrm{~g}$ of alcohol per days and $60 \%$ of the subjects were consuming over a period of 10-14 years.

4. $40 \%$ of the subjects were observed to have hypoalbuminemia, $28 \%$ of the subjects had hyperglobulinemia; the percentage of patients with elevated AST,ALT, ALP, bilirubin and prolonged prothrombin time were $42 \%, 8 \%, 12 \%, 40 \%$ and $28 \%$ respectively. $76 \%$ of the subjects had the ratio of AST to ALT more than 1 with $40 \%$ of the subjects having the ratio more than 2 .

5. There was a statistically significant positive correlation between quantity of alcohol consumption and serum levels of globulin, SGPT, bilirubin and prothrombin time. Where as for serum albumin levels there was a negative correlation.

6. There was statistically significant correlation between duration of alcohol consumption with serum albumin and bilirubin levels, negatively for albumin and positively for bilirubin. 


\section{References}

[1] Sheila Sherlock, James Dooley. Alcohol and the liver. Chapter 22. Diseases of the liver and biliary system. Blackwell Science publications. $11^{\text {th }}$ ed.2002;381-396.

[2] Rehm J, Mathers C, Popova S, Thavorncharoensap M, Teerawattananon Y, Patra J. Global burden of disease and injury and economic cost attributable to alcohol use and alcohol-use disorders. Lancet. 2009;373:2223-2233

[3] Maher JJ. Exploring alcohol's effects on liver function. Alcohol Health Res World.1997;21:5-12

[4] Usharani B, Vennila R, Nalini N. Biochemical changes in alcoholics- A case control study. International journal of research in pharmaceutical and biomedical sciences.2012;3:201-205.

[5] Das SK, Vasudevan DM. Biochemical diagnosis of alcoholism. Indian J Clin Biochem. 2005;20:35-42

[6] Cohen JA, Kaplan M M. The SGOT/SGPT ratio-an indicator of alcoholic liver disease. Digestive Diseases and Sciences.1979;24:835-838.

[7] Van Waes L, Leiber CS. Glutamate dehydrogenase: a reliable marker of liver cell necrosis in the alcoholic. Br Med J.1977;2:150810

[8] Madenhall CL, Moritz TE. A study of oral nutritional support with oxandrolone in malnourished patients with alcoholic hepatitis. Hepatology. 1993; 17:564 